\title{
Correlated Post-mortem Raman and TEM Investigation of Nanoindentation Induced Structural Changes in Silicon as a Function of Temperature
}

Praveena Manimunda ${ }^{1}$, Eric Hintsala ${ }^{1}$, Douglas Stauffer ${ }^{1}$ and S.A.S. Asif ${ }^{2}$

${ }^{1}$ Bruker Nano Surfaces, Eden Prairie, Minnesota, United States, ${ }^{2}$ Industron, Thiruvananthapuram, Kerala, India

Silicon remains one of the most technologically important materials, seeing use in a variety of microelectronics and Micro-Electro-Mechanical Systems (MEMS) devices and sensors. Several decades of intense industrial research have led to some of the most sophisticated materials processing routes for Silicon, yet some of the details regarding its mechanical properties remain mysterious. This is not for a lack of effort, but rather due to its complexity. In terms of deformation mechanisms, dislocation plasticity, fracture and various phase transformations are all possible depending on the loading rate, the state of stress, size, temperature, the presence of impurities and more. For this study, focus was made on the phase transformations in Silicon, which occur under confining pressure in predominantly compressive loadings [1-3]. This makes instrumented indentation a popular choice for inducing such behaviors [4,5], which we have done here at various temperatures. What is unique is in this study is correlated use of two postmortem microscopy techniques: Raman mapping of the indents and transmission electron microscopy (TEM) of focused ion beam (FIB) machined liftouts. This was done to try to understand in greater detail not only which phase transformations are occurring, but how they are spatially distributed and this relation to the state of stress locally underneath the indenter. Silicon wafers with $<001>$ orientation and p-type doped to a resistivity of 0.001-0.005 $\Omega$-cm, corresponding to 1x1019 - 1x1020 cm-3 boron doping were tested at elevated temperatures using both a 800C equipped Hysitron PI88 in situ SEM indenter and a prototype high vacuum platform nanoindenter system both equipped with a diamond Berkovich tip. Raman mapping was carried out using a Witec Alpha 300R Confocal Raman Microscope. Lift-outs were prepared along the diagonal of an indent, thus bisecting one face and one corner. Protective platinum was deposited prior to thinning and transfer to half grid. Sample preparation was done with an FEI Versa 3D dualbeam and EasyLift manipulator (Thermo Fischer Scientific, Hillsboro) and imaged using a Technai F30 TEM operating at $300 \mathrm{keV}$ in bright field. A summary of the changes in the nanoindentation behaviour for Silicon from room temperature to $450^{\circ} \mathrm{C}$ is shown in Figure 1. Here, the hardness rises initially with temperature up to roughly $150^{\circ} \mathrm{C}$, before starting to steadily decrease. This is a rather interesting observation, as generally hardness and yield strength decrease with increasing temperature when the properties are mediated by dislocation plasticity - this indicates that other behaviour is dominant in the low temperature regime. This is also demonstrated in the post-mortem SEM imaging of the indents, as at room temperature there is spalling, which disappears at $100^{\circ} \mathrm{C}$, then becomes ductile flow at $200^{\circ} \mathrm{C}$. The spallation is attributed to phase transformations during unloading where the lattice expands. Some understanding of the deformation mechanism changes that explain this change in hardness are demonstrated in Figure 2, where post-mortem Raman maps and TEM images are shown. Here, the room temperature Raman map shows a strongly phase transformed region under the indenter impression which is also apparent from the TEM imaging. As the temperature is increased to $100^{\circ} \mathrm{C}$, the Raman spectra shows a dramatic shift from a complex mixture of amorphous, R8 and BC8 Silicon phases to hexagonal and diamond cubic phases. The postmortem TEM also shows a change in the phase transformed region, particularly in the reduction in total phase transformed material. At $200^{\circ} \mathrm{C}$, diamond cubic is shown in the Raman spectra, with a small amount of hexagonal material. The TEM shows the dominance of what appears to be twinning based plasticity under the indent with little apparent phase transformed material. 

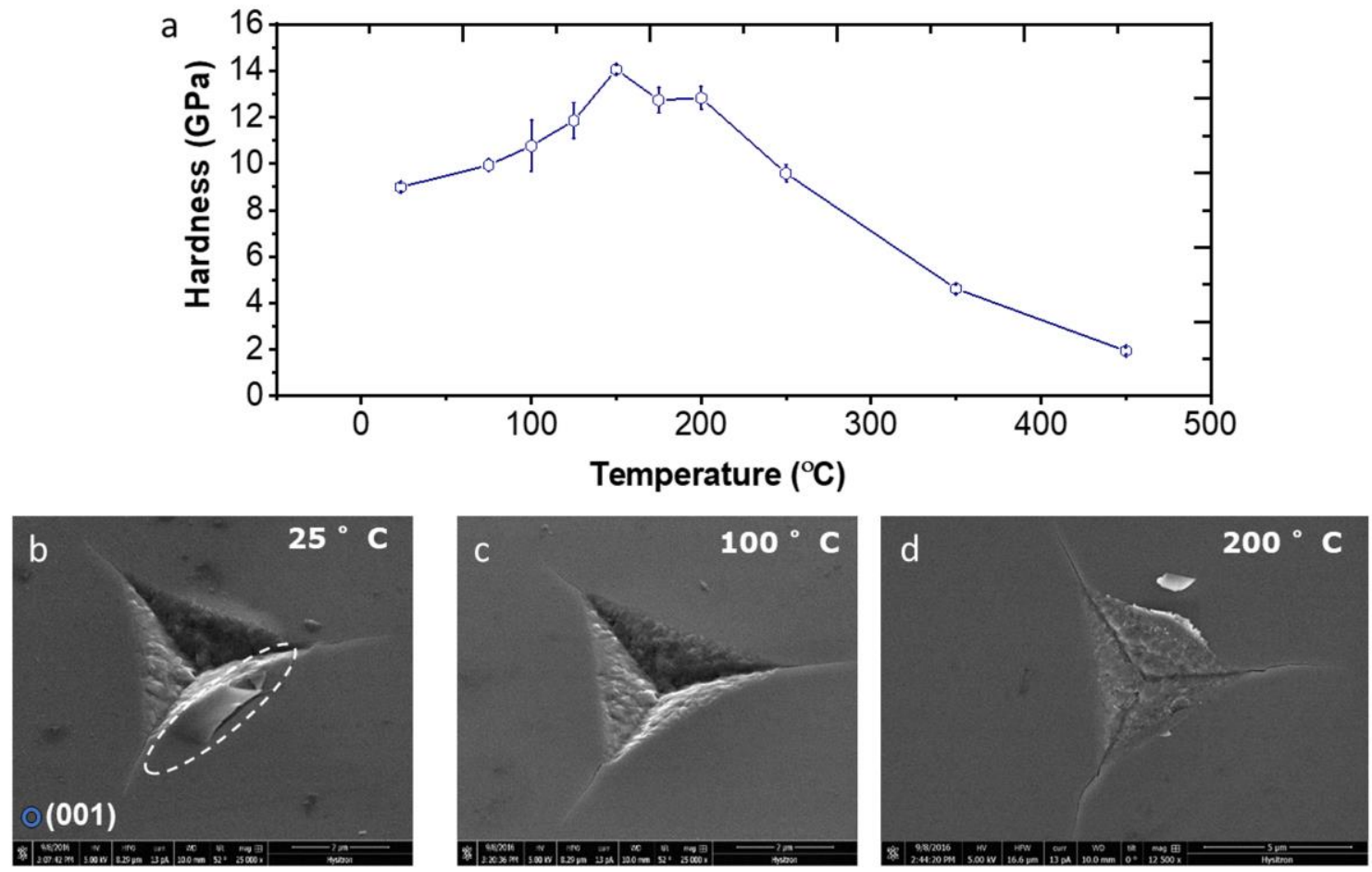

Figure 1. a) Hardness versus temperature for $<100>$ Silicon and (b-d) post-mortem SEM images showing change in behavior from spallation to plasticity

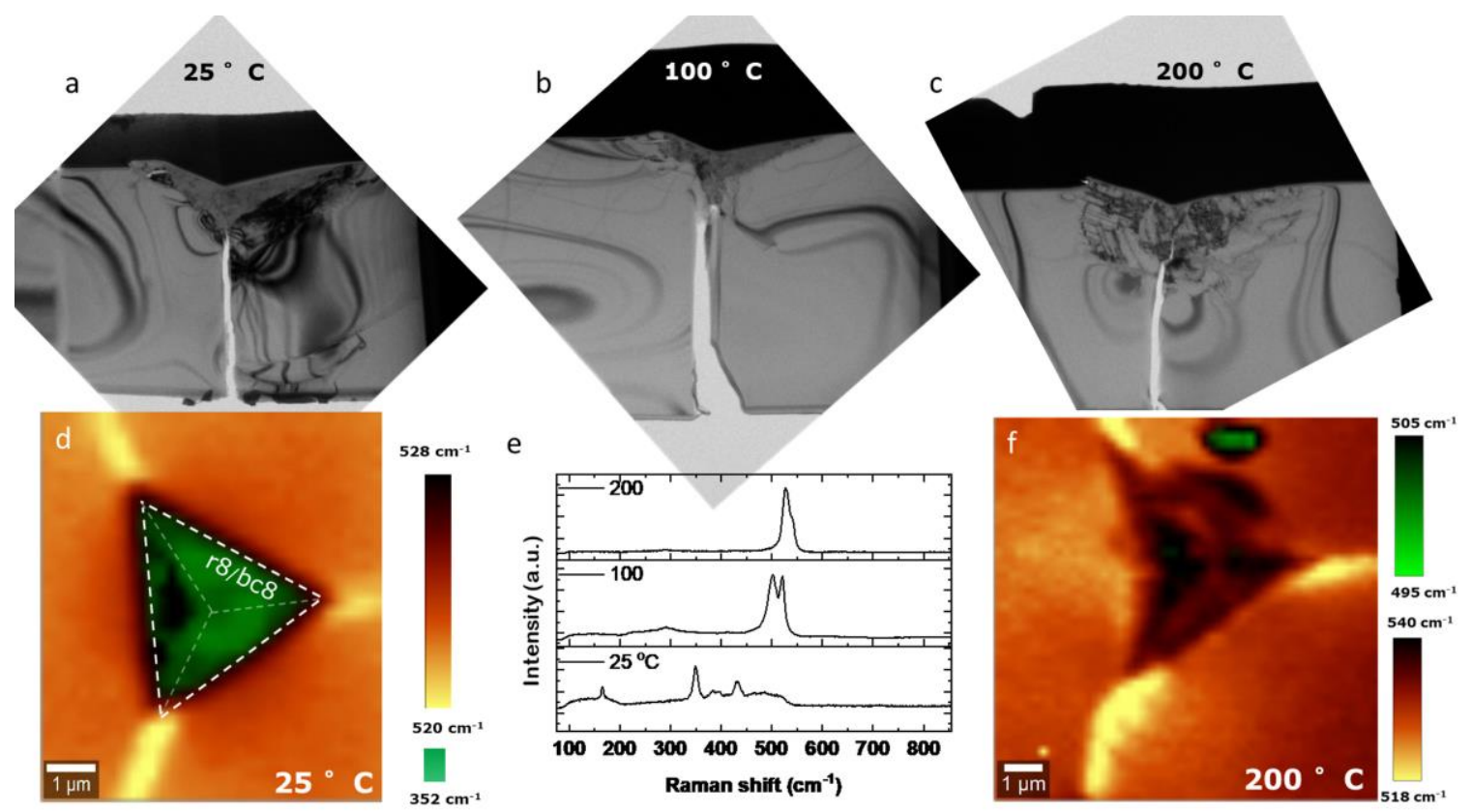

Figure 2. (a-c) Post-mortem TEM of FIB prepared liftouts from room temperature to 200C. (d,f) Corresponding raman maps of room temperature and $200 \mathrm{C}$ indents and e) overall spectra of the three test temperatures. 


\section{References}

[1] Weinstein, B. A., \& Piermarini, G. J. (1975). Raman scattering and phonon dispersion in Si and GaP at very high pressure. Physical Review B, 12(4), 1172.

[2] Minomura, S., \& Drickamer, H. G. (1962). Pressure induced phase transitions in silicon, germanium and some III-V compounds. Journal of Physics and Chemistry of Solids, 23(5), 451-456.

[3] Jamieson, J. C. (1963). Crystal structures at high pressures of metallic modifications of silicon and germanium. Science, 139(3556), 762-764.

[4] Gerbig, Y. B., Michaels, C. A., Forster, A. M., \& Cook, R. F. (2012). In situ observation of the indentation-induced phase transformation of silicon thin films. Physical Review B, 85(10), 104102.

[5] Domnich, V., Aratyn, Y., Kriven, W. M., \& Gogotsi, Y. (2008). Temperature dependence of silicon hardness: experimental evidence of phase transformations. Rev. Adv. Mater. Sci, 17(1-2), 33-41. 